\title{
Pure Laparoscopic Right Upper Transversal Hepatectomy
}

\author{
Fei Liu, MD, YongGang Wei, MD, and Bo Li, MD
}

Department of Liver Surgery \& Liver Transplantation Center, West China Hospital, Sichuan University, Chengdu, Sichuan Province, China

\begin{abstract}
Background. First proposed by Torzilli et al., ${ }^{1}$ the right upper transversal hepatectomy (RUTH) was one of the most complex techniques of parenchymal-sparing hepatectomies. Moreover, this operation is technically challenging, and studies were limited to case series. ${ }^{2-4}$ Nevertheless, this complicated procedure has not been reported to perform laparoscopically. We present a video of pure laparoscopic RUTH.
\end{abstract}

Methods. A 36-year-old female with HBV-related hepatocellular carcinoma (HCC) was admitted to our institution. Abdominal CT showed a 5.5- $\times 5.0-\mathrm{cm}$ mass located in deep S7-8 with involvement of middle hepatic vein (MHV) and right hepatic vein (RHV). Extended right hepatectomy was not adopted because of the insufficient volume of residual liver. Moreover, a thick inferior right hepatic vein (IRHV) and two communicating veins (CVs) were observed by preoperative 3-D reconstruction. Thus, laparoscopic RUTH was performed.

Results. After fully mobilization of right liver, the RHV and MHV were isolated and encircled. Then, intraoperative ultrasound was used to locate the tumor and identify the tumor-bearing portal territories. Subsequently, parenchymal transection was performed using the Harmonic. The Pringle's maneuver was used intermittently during transection as necessary (total Pringle time was $62 \mathrm{~min}$ ). Four tumor-bearing portal pedicles were all confirmed and divided with the guidance of intraoperative ultrasound. The

(C) Society of Surgical Oncology 2022

First Received: 13 September 2021

Accepted: 3 January 2022;

Published Online: 22 January 2022

Y. Wei, MD

e-mail: hxwyg@scu.edu.cn

B. Li, MD

e-mail: cdlibo688@163.com
MHV and RHV were transected using the stapler and the IRHV was well preserved. Finally, the operation was finished and the color of residual liver was acceptable. The operative time was $240 \mathrm{~min}$ and estimated blood loss was $100 \mathrm{cc}$. The postoperative course was uneventful and the patient was discharged on postoperative Day 6 .

Conclusions. Laparoscopic RUTH is technically feasible and safe in selected patients with IRHV and CVs.

Supplementary Information The online version contains supplementary material available at https://doi.org/10.1245/s10434022-11331-8.

DISCLOSURES Drs. Fei Liu, YongGang Wei, and Bo Li have no conflict of interest or financial ties to disclose.

\section{REFERENCES}

1. Torzilli G, Procopio F, Donadon M, et al. Upper transversal hepatectomy. Ann Surg Oncol. 2012;19(11):3566.

2. Torzilli G, Procopio F, Cimino M, et al. Radical but conservative liver resection for large centrally located hepatocellular carcinoma: the mini upper-transversal hepatectomy. Ann Surg Oncol. 2014;21(6):1852.

3. Alvarez FA, Sanchez Claria R, Oggero S, de Santibañes E. Parenchymal-sparing liver surgery in patients with colorectal carcinoma liver metastases. World $J$ Gastrointest Surg. 2016;8(6):407-23.

4. Makdissi FF, Kruger JAP, Jeismann VB, Herman P. Feasibility of right upper transversal hepatectomy in the absence of an inferior right hepatic vein: new insights regarding this complex procedure. Case Rep Surg. 2021;2021:6668269.

Publisher's Note Springer Nature remains neutral with regard to jurisdictional claims in published maps and institutional affiliations. 